\title{
Improved Pilot-Aided Channel Estimation for MIMO-OFDM Fading Channels
}

\author{
J. Mar, ${ }^{1,2}$ Chi-Cheng Kuo, ${ }^{1}$ and M. B. Basnet ${ }^{1}$ \\ ${ }^{1}$ Department of Communications Engineering, Yuan-Ze University, 135 Yuan-Tung Road, Jungli, Taoyuan 320, Taiwan \\ ${ }^{2}$ Communications Research Center, Yuan-Ze University, 135 Yuan-Tung Road, Jungli, Taoyuan 320, Taiwan \\ Correspondence should be addressed to J. Mar; eejmar@saturn.yzu.edu.tw
}

Received 2 July 2013; Accepted 11 August 2013

Academic Editor: Ai Bo

Copyright (c) 2013 J. Mar et al. This is an open access article distributed under the Creative Commons Attribution License, which permits unrestricted use, distribution, and reproduction in any medium, provided the original work is properly cited.

\begin{abstract}
An improved pilot-aided channel estimation scheme is proposed to enhance the channel estimation accuracy of multiple-input multiple-output-orthogonal frequency division multiplexing (MIMO-OFDM) fading channels. Based on the adaptive path number selection mechanism, the number of paths can be scalable and adaptively changed with the characteristics of MIMO-OFDM fading channels. The fine channel estimation formulas for all data subcarriers are derived. The $2 \times 2$ space-frequency block code-OFDM (SFBC-OFDM) system and a six-path fading channel model are considered as an example of the high mobility MIMO-OFDM wireless communications system. Through simulations it is shown that $2 \times 2$ SFBC-OFDM system using the proposed approach can satisfy the performance requirements over frequency selective and frequency nonselective fast fading channels.
\end{abstract}

\section{Introduction}

The cellular mobile communications industry has recently been one of the fastest growing industries of all time, with the number of users increasing incredibly rapidly. Orthogonal frequency division multiplexing access (OFDMA) was chosen as the spectrum access technology of the 4G cellular systems because its orthogonality eliminates intracell interference. The high mobility OFDM wireless communication system will operate in a fast fading channel, where the nonnegligible fluctuations of the channel gains are expected within each OFDM data block. Fast fading involves variations on the scale of a half-wavelength and frequently introduces variations as large as $35-40 \mathrm{~dB}$ [1]. The channel estimation in OFDM systems over time-varying fading channels is generally based on the use of pilot subcarriers in given positions of the frequency-time grid [2]. It is advisable to place pilot subcarriers in each OFDM data block in order to ensure adequate estimation accuracy. In [3], the effect of pilot power on the performance of 16-QAM OFDM system operating in two-ray Rayleigh slow fading channel is presented. The optimum pilot-to-data power ratio (PDR) is analytically derived. As an alternative, the channel estimation algorithm based on subspace tracking has been presented in
[4] for OFDM systems, which can effectively reduce channel estimation error by tracking the dominant delay-subspace spanned by the frequency responses.

Increasing demand for high performance $4 \mathrm{G}$ broadband wirelesses is enabled by the use of multiple antennas at both base station and user equipment ends. Multiple-inputmultiple-output (MIMO) is one of the best ways to combat channel fading using transmit diversity and receive diversity. The use of MIMO technique in OFDM system is an efficient solution to meet the growing demand for high speed, spectral efficiency, and reliable communication [2] in future-generation wireless networks. The MIMO-OFDM wireless communications have the inherent signal variability generated from the multipath fading channel. The aim of this study is to investigate the channel estimation algorithm in the MIMO-OFDM fading channels. In [5], it is shown that the space-frequency block code-(SFBC-) OFDM system exhibits error floors caused by imperfect channel state estimation over frequency selective fading channels. Hence, we need a more robust frequency and phase synchronization technology for fast MIMO-OFDM fading channels. The optimum pilot allocation in terms of overhead and channel estimation error is analyzed in reference [6] that maximizes the channel 
capacity for MIMO-OFDM system operating in frequency selective fading channel. Both perfect interpolation and nonperfect interpolation for pilot-aided channel estimations are considered. In [7], the sequential decision feedback sequence estimation with an adaptive threshold equalizer technique and pilot tone plus interpolation channel estimation scheme are used to design the Alamouti coded small constellation (BPSK and QPSK) OFDM receiver in fast fading channels.

An adaptive path number selection mechanism is proposed for channel estimation over MIMO-OFDM fading channels to provide the suboptimum system performance, whenever the high order modulation MIMO-OFDM system is operated either in frequency nonselective fast fading or in frequency selective fast fading channels. The $2 \times 2$ SFBCOFDM system and a six-path fading channel model are considered as an example of the MIMO-OFDM system in the simulations to prove that the acceptable bit error rate (BER) can be achieved by employing 16-QAM and 64QAM modulations in time-varying fast fading channels. We consider the vehicle speed of $200 \mathrm{~km} / \mathrm{h}$, resulting in the Doppler frequency of $1093 \mathrm{~Hz}$ to satisfy the fast fading condition for MIMO-OFDM channel $[8,9]$.

The rest of this paper is organized as follows. The proposed MIMO-OFDM channel estimation algorithm is described in Section 2, where the fine channel estimation for all data subcarriers is derived. The adaptive path number selection mechanism for the MIMO-OFDM fading channel is presented in Section 3. The BER performance of the MIMO-OFDM system using the proposed suboptimal channel estimation approach is simulated and discussed in Section 4. Finally, concluding remarks are given in Section 5.

\section{MIMO-OFDM Channel Estimation}

We consider a generic downlink multiuser MIMO-OFDM channel model. Let the number of transmit antennas be $n_{T}$ and the number of receive antennas $n_{R}$. One OFDM symbol of each user is transmitted across $N$ subcarriers. To simplify the formula derivations, the data vector $\mathbf{X}$ for each user can be expressed in polyphase representation as

$$
\mathbf{X}=\left[\begin{array}{lllll}
X_{0} & X_{1} & \cdots & X_{N-2} & X_{N-1}
\end{array}\right]^{T},
$$

where $T$ denotes the transpose of the vector. Thus, the demodulated signal vector is given by

$$
\mathbf{Y}=\mathbf{H X}+\mathbf{V}
$$

where $\mathbf{H}$ is a diagonal matrix whose diagonal elements are the $N$-DFT of the channel impulse response $\mathbf{h}$ and $\mathbf{V}$ is the $N$-DFT of the channel noise. With reference to the conventional channel estimation approach of a given OFDM system [9], ten short OFDM training signals are used for packet detection, coarse frequency offset estimation, and timing synchronization. Two periods of the long training signals are used for improving channel estimation accuracy of the short training symbols. A phase-locked loop is adopted in the receiver for estimating and compensating the carrier frequency offset. Each OFDM data block contains $L$ pilot subcarriers, which are used to track the carrier phase.
A typical $2 \times 2$ SFBC-OFDM model, which consists of two transmit antennas and two receive antennas, is used to describe the theoretical analysis and the proposed channel estimation scheme. User data vector $\mathbf{X}$ is first encoded into two spatial vectors $\mathbf{X}_{1}$ and $\mathbf{X}_{2}$ by the space-frequency encoder. Denote the transmitted signal vector of each user in a space-frequency block as

$$
\begin{gathered}
\mathbf{X}_{1}=\left[\begin{array}{lllll}
X_{0} & -X_{1}^{*} & \cdots & X_{N-2} & -X_{N-1}^{*}
\end{array}\right]^{T}, \\
\mathbf{X}_{2}=\left[\begin{array}{lllll}
X_{1} & X_{0}^{*} & \cdots & X_{N-1} & X_{N-2}^{*}
\end{array}\right]^{T},
\end{gathered}
$$

where $\mathbf{X}_{1}$ is the data transmitted from the first antenna $T x_{1}$ and $\mathbf{X}_{2}$ is the data transmitted from the second antenna $T x_{2}$, simultaneously. Let $\mathbf{X}_{e}$ and $\mathbf{X}_{o}$ be even and odd component vectors of $\mathbf{X}$; that is,

$$
\begin{aligned}
& \mathbf{X}_{e}=\left[\begin{array}{lllll}
X_{0} & X_{2} & \cdots & X_{N-4} & X_{N-2}
\end{array}\right]^{T}, \\
& \mathbf{X}_{o}=\left[\begin{array}{lllll}
X_{1} & X_{3} & \cdots & X_{N-3} & X_{N-1}
\end{array}\right]^{T} .
\end{aligned}
$$

Similarly, $\mathbf{X}_{1, e}, \mathbf{X}_{1, o}, \mathbf{X}_{2, e}$, and $\mathbf{X}_{2, o}$ denote even and odd component vectors of $\mathbf{X}_{1}$ and $\mathbf{X}_{2}$, respectively, which can then be expressed in terms of even and odd component vectors as

$$
\begin{array}{ll}
\mathbf{X}_{1, e}=\mathbf{X}_{e}, & \mathbf{X}_{1, o}=-\mathbf{X}_{o}^{*}, \\
\mathbf{X}_{2, e}=\mathbf{X}_{o}, & \mathbf{X}_{2, o}=\mathbf{X}_{e}^{*} .
\end{array}
$$

Note that since the two corresponding signals transmitted from two antennas at the same time slots are orthogonal, the maximum likelihood decoding is reduced to simple linear processing at the receiver. The received signal at the receiver is given by

$$
\begin{aligned}
& \mathbf{Y}_{1}=\mathbf{H}_{1,1} \mathbf{X}_{1}+\mathbf{H}_{2,1} \mathbf{X}_{2}+\mathbf{V}_{1}, \\
& \mathbf{Y}_{2}=\mathbf{H}_{1,2} \mathbf{X}_{1}+\mathbf{H}_{2,2} \mathbf{X}_{2}+\mathbf{V}_{2},
\end{aligned}
$$

where $\mathbf{Y}_{1}$ and $\mathbf{Y}_{2}$ are the received signals in the first and second received antenna, $\mathbf{H}_{1,1}$ and $\mathbf{H}_{2,1}$ are the channel frequency response of the first and second antenna transmitted to the first received antenna, and $\mathbf{H}_{1,2}$ and $\mathbf{H}_{2,2}$ are the channel frequency response of the first and second antenna transmitted to the second received antenna. The channel frequency response at all data subcarriers for each transmitreceive antenna pair is defined as

$$
\begin{gathered}
\mathbf{H}_{p, q}=\left[\begin{array}{cccc}
H_{p, q, 0} & 0 & \ldots & 0 \\
0 & H_{p, q, 1} & \ldots & 0 \\
\ldots & \ldots & \ldots & \ldots \\
0 & 0 & \ldots & H_{p, q, N-1}
\end{array}\right] \\
\text { for }\left\{\begin{array}{l}
p=1, \ldots, n_{T} \\
q=1, \ldots, n_{R}
\end{array} .\right.
\end{gathered}
$$

Equivalently, (6) can be represented as

$$
\begin{aligned}
& \mathbf{Y}_{1, e}=\mathbf{H}_{1,1, e} \mathbf{X}_{1, e}+\mathbf{H}_{2,1, e} \mathbf{X}_{2, e}+\mathbf{V}_{1, e}, \\
& \mathbf{Y}_{1, o}=\mathbf{H}_{1,1, o} \mathbf{X}_{1, o}+\mathbf{H}_{2,1, o} \mathbf{X}_{2, o}+\mathbf{V}_{1, o}, \\
& \mathbf{Y}_{2, e}=\mathbf{H}_{1,2, e} \mathbf{X}_{1, e}+\mathbf{H}_{2,2, e} \mathbf{X}_{2, e}+\mathbf{V}_{2, e}, \\
& \mathbf{Y}_{2, o}=\mathbf{H}_{1,2, o} \mathbf{X}_{1, o}+\mathbf{H}_{2,2, o} \mathbf{X}_{2, o}+\mathbf{V}_{2, o} .
\end{aligned}
$$




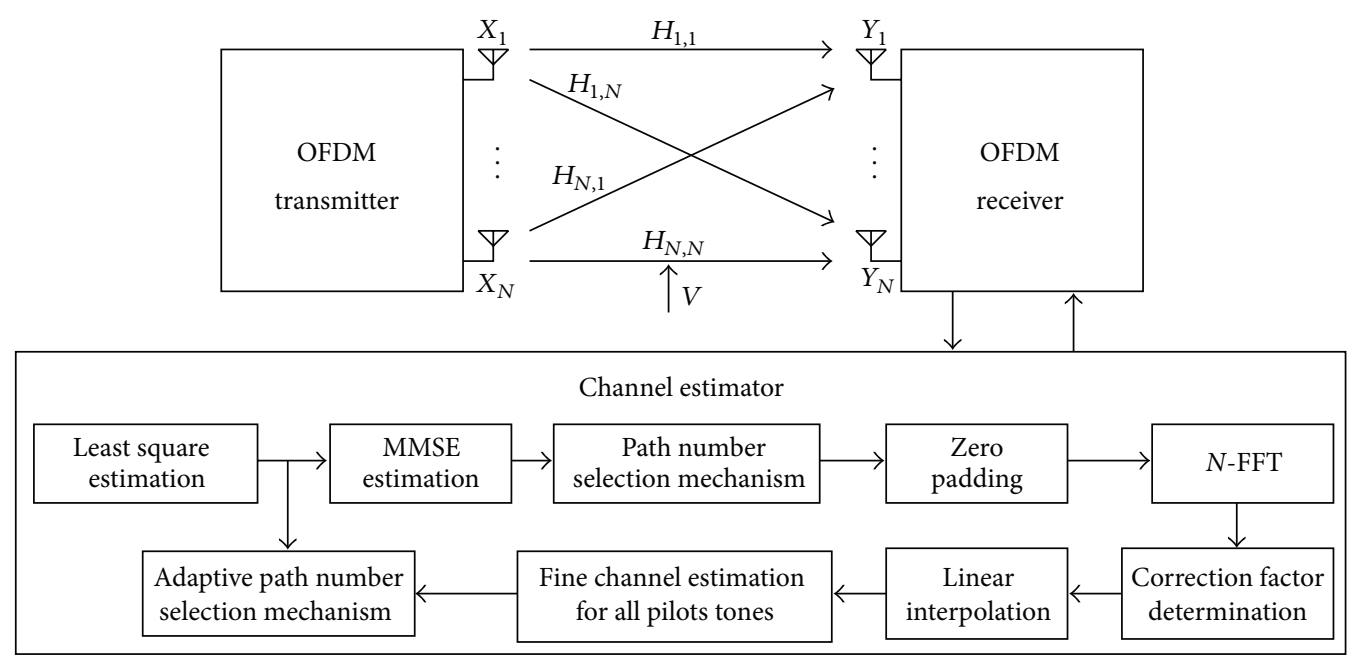

FIGURE 1: Block diagram of pilot tone channel estimation aided with adaptive path number selection mechanism for MIMO-OFDM fading channel.

The received signal at the $q$ th receive antenna for the $k$ th pilot tone transmitted from $p$ th antenna can be written as

$$
\begin{aligned}
Y_{q, k}=X_{p, k} H_{p, q, k}+V_{q, k}, \quad k & =0,1, \ldots, N-1, \\
p & =1, \ldots, n_{T}, \\
q & =1, \ldots, n_{R},
\end{aligned}
$$

where $N$ is the frequency tones in each OFDM data block, $X_{p, k}$ is the transmitted signal of $p$ th transmitted antenna, $H_{p, q, k}$ is the channel frequency response form $p$ th transmit antenna to $q$ th receive antenna, and $V_{q, k}$ is the AWGN noise.

Then from (9), the channel estimation at pilot subcarriers based on the least square (LS) algorithm can be obtained as

$$
\widehat{H}_{p, q, k}=\frac{Y_{q, k}}{X_{p, k}}=H_{p, q, k}+\frac{S_{q, k}}{\sqrt{\varepsilon_{x}}}, \quad k=0,1, \ldots, N-1,
$$

where $S_{q, k}=V_{q, k} / e^{j \angle X_{m, k}},\left|X_{p, k}\right|=\sqrt{\varepsilon_{x}}$, and $X_{p, k}=\left|X_{p, k}\right| e^{j \angle X_{p, k}}$. Let $\left\{k_{1}, k_{2}, \ldots, k_{L}\right\}$ be the set of $L$ pilot tones, which is one of the sets $\{i, i+N / L, \ldots, i+(L-1) N / L\}, i=0,1, \ldots, N / L-1$, used for transmitting the training data. Collect these channel responses in a vector $\widehat{\mathbf{H}}_{p, q, k_{p}}=\left[\widehat{H}_{p, q, k_{1}}, \ldots, \widehat{H}_{p, q, k_{L}}\right]^{T}$, which is obtained from the FFT matrix.

The intermediate processing steps between the LS estimates of the channel gains over the pilot subcarriers and interpolation processing are added in order to ensure adequate estimation accuracy for fast fading channel. The block diagram of the proposed pilot tone channel estimation aided with adaptive path number selection mechanism is shown in Figure 1. Here $\ell$ is defined as the number of dominant paths estimated from the adaptive channel path number selector, which chooses $\ell$ paths with larger power from $\left(\widehat{\mathbf{h}}_{p, q, k_{p}}\right)_{L \times 1}$, and let $\left\{b_{1}, b_{2}, \ldots, b_{\ell}\right\}$ be a set of the selected pilot index. Since
AWGN assumption for each subcarrier is adopted, and since each pilot tone carries data of constant modulus $\sqrt{\varepsilon_{x}}$, the minimum mean square error (MMSE) estimation of $\mathbf{h}_{p, q}$ is given by [10]

$$
\begin{aligned}
\widehat{\mathbf{h}}_{p, q, k_{p}} & =\mathbf{Q}_{p, q, k_{p}}^{-1} \widehat{\mathbf{H}}_{p, q, k_{p}} \\
& =\mathbf{h}_{p, q, k_{p}}+\mathbf{Q}_{p, q, k_{p}}^{-1} \frac{1}{\sqrt{\varepsilon_{x}}} \mathbf{S}_{k_{p}},
\end{aligned}
$$

where $\mathbf{S}_{q, k_{p}}=\left[S_{q, k_{1}}, \ldots, S_{q, k_{L}}\right]^{T},\left(\widehat{\mathbf{h}}_{p, q, k_{p}}\right)_{\ell \times 1}=\left[\widehat{h}_{p, q, k_{1}} \widehat{h}_{p, q, k_{2}}\right.$ $\left.\cdots \widehat{h}_{p, q, k_{\ell}}\right]^{T}$, and $\mathbf{Q}_{p, q, k_{p}}$ is a Vandermonde matrix with distinct $L$ twiddle factor $W_{N}^{k_{i}}$ :

$\left(\left(\mathbf{Q}_{p, q, k_{p}}\right)_{\ell \times L}\right)^{-1}$

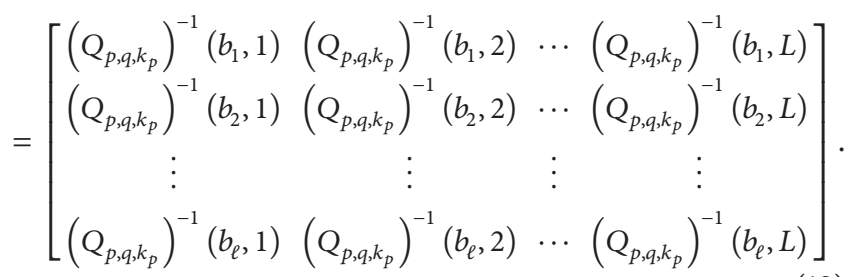

Other notations are represented as follows: $E\{\cdot\}$ is the expectation operator; $\operatorname{tr}\{\cdot\}$ is the trace operator; $\|\cdot\|$ means 2-norm; $\mathbf{I}_{L}$ represents the $L \times L$ identity matrix. Therefore, the mean square error (MSE) in the channel estimate can be derived as

$$
E\left\{\left\|\left(\widehat{\mathbf{h}}_{p, q}\right)_{\ell \times 1}-\left(\mathbf{h}_{p, q}\right)_{\ell \times 1}\right\|^{2}\right\}=\frac{\sigma_{s}^{2}}{\varepsilon_{x}} \operatorname{tr}\left\{\left(\mathbf{Q}_{p, q, k_{p}}^{*} \mathbf{Q}_{p, q, k_{p}}\right)_{\ell \times \ell}^{-1}\right\},
$$


where $\sigma_{s}^{2}$ is the variance of AWGN which is assumed to be known at the receiver, and

$$
\begin{aligned}
& \left(\mathbf{Q}_{p, q, k_{p}}^{*} \mathbf{Q}_{p, q, k_{p}}\right)_{\ell \times \ell}^{-1} \\
& =\left[\begin{array}{ccccc}
\left(\mathbf{Q}_{p, q, k_{p}}^{*} \mathbf{Q}_{p, q, k_{p}}\right)^{-1} & \left(b_{1}, b_{1}\right) & \cdots & \left(\mathbf{Q}_{p, q, k_{p}}^{*} \mathbf{Q}_{p, q, k_{p}}\right)^{-1} & \left(b_{1}, b_{\ell}\right) \\
\left(\mathbf{Q}_{p, q, k_{p}}^{*} \mathbf{Q}_{p, q, k_{p}}\right)^{-1} & \left(b_{2}, b_{1}\right) & \cdots & \left(\mathbf{Q}_{p, q, k_{p}}^{*} \mathbf{Q}_{p, q, k_{p}}\right)^{-1} & \left(b_{2}, b_{\ell}\right) \\
\vdots & \ldots & & \vdots & \\
\left(\mathbf{Q}_{p, q, k_{p}}^{*} \mathbf{Q}_{p, q, k_{p}}\right)^{-1} & \left(b_{\ell}, b_{1}\right) & \cdots & \left(\mathbf{Q}_{p, q, k_{p}}^{*} \mathbf{Q}_{p, q, k_{p}}\right)^{-1} & \left(b_{\ell}, b_{\ell}\right)
\end{array}\right],
\end{aligned}
$$

where $(\cdot)^{*}$ denotes the Hermitian transposition and $\mathbf{Q}_{p, q, k_{p}}$ depends on the choice of the set of pilot tones. Let the eigenvalues of $\left(\mathbf{Q}_{p, q, k_{p}}^{*} \mathbf{Q}_{p, q, k_{p}}\right)_{\ell \times \ell}^{-1}$ be denoted by $\left\{\lambda_{m}, m=0,1\right.$, $\ldots, \ell-1\}$. Then, the eigenvalues of $\left(\mathbf{Q}_{p, q, k_{p}}^{*} \mathbf{Q}_{p, q, k_{p}}\right)_{\ell \times \ell}$ are $\left\{1 / \lambda_{m}, m=0,1, \ldots, \ell-1\right\}$. Since the trace of a matrix is the sum of its eigenvalues, then

$$
\begin{aligned}
& \operatorname{tr}\left\{\left(\mathbf{Q}_{p, q, k_{p}}^{*} \mathbf{Q}_{p, q, k_{p}}\right)_{\ell \times \ell}\right\}=\sum_{m=0}^{\ell-1} \frac{1}{\lambda_{m}}=\frac{L}{N} \ell, \\
& \operatorname{tr}\left\{\left(\mathbf{Q}_{p, q, k_{p}}^{*} \mathbf{Q}_{p, q, k_{p}}\right)_{\ell \times \ell}^{-1}\right\}=\sum_{m=0}^{\ell-1} \lambda_{m}=\frac{N}{L} \ell .
\end{aligned}
$$

Substituting (16) into (13) yields the MMSE in channel estimate, when such a pilot tone set is used:

$$
E\left\{\left\|\left(\widehat{\mathbf{h}}_{p, q}\right)_{\ell \times 1}-\left(\mathbf{h}_{p, q}\right)_{\ell \times 1}\right\|^{2}\right\}=\frac{\sigma_{s}^{2} N \ell}{\varepsilon_{x} L} .
$$

If $\ell=L$, then MMSE is

$$
E\left\{\left\|\widehat{\mathbf{h}}_{p, q}-\mathbf{h}_{p, q}\right\|^{2}\right\}=\frac{\sigma_{s}^{2} N}{\varepsilon_{x}} .
$$

Equations (17) and (18) show that using less pilot number in the pilot-aided channel estimation can get smaller MSE in the channel impulse response estimate. However, in the frequency selective fast fading channel, less pilot number may cause more linear interpolation loss. The case of $\ell=1$ will not be considered for path number selection mechanism because it cannot reflect the variation of channel characterization of the frequency selective fading channel. The preliminary channel frequency response estimate $\left(\widetilde{\mathbf{H}}_{p, q}\right)_{N \times 1}$ is obtained by the $N$-FFT of an $N \times 1$ estimated channel impulse response $\left(\widehat{\mathbf{h}}_{p, q}^{\ell}\right)_{N \times 1}=\left[\left(\widehat{\mathbf{h}}_{p, q}^{T}\right)_{\ell \times 1} \mathbf{0}_{(N-\ell) \times 1}^{T}\right]^{T}$. It can be written as

$$
\widetilde{H}_{p, q, k}^{\ell}=\frac{1}{\sqrt{N}} \sum_{n=0}^{\ell-1} \widehat{h}_{p, q}^{\ell}(n) W_{N}^{k n}, \quad k=0,1, \ldots, N-1 .
$$

The preliminary channel frequency response estimates at pilot tones for $k=k_{1}, k_{2}, \ldots, k_{L}$ are $\widetilde{\mathbf{H}}_{p, q, L \times 1}^{\ell}=\left[\widetilde{H}_{p, q, k_{1}}^{\ell}, \ldots\right.$, $\left.\widetilde{H}_{p, q, k_{L}}^{\ell}\right]^{T}$. The correction factor for fine channel frequency response estimate at $k_{p}$ th pilot tone is defined as

$$
C_{p, q, k_{p}}^{\ell}=\frac{\widehat{H}_{p, q, k_{p}}}{\widetilde{H}_{p, q, k_{p}}^{\ell}}=\frac{(1 / \sqrt{N}) \sum_{n=0}^{L-1} \widehat{h}_{p, q}(n) W_{N}^{k_{p} n}}{(1 / \sqrt{N}) \sum_{n=0}^{\ell-1} \widehat{h}_{p, q}^{\ell}(n) W_{N}^{k_{p} n}} .
$$

For example, the fine correction factor at $k_{1}$ th pilot tone for $L=4$ and $\ell=2$ is determined as

$$
C_{p, q, k_{1}}^{2}=\frac{\widehat{H}_{p, q, k_{1}}}{\widetilde{H}_{p, q, k_{1}}^{2}}=1+\frac{\widehat{h}_{p, q}(2) W_{N}^{2 k_{1}}+\widehat{h}_{p, q}(3) W_{N}^{3 k_{1}}}{\widehat{h}_{p, q}(0)+\widehat{h}_{p, q}(1) W_{N}^{k_{1}}} .
$$

From (21), it is observed that the fine correction factor can compensate the power loss caused by less path employed in the preliminary channel estimates. When the number of paths chosen is $\ell$, the fine correction factor in a vector for $L$ pilot tones is

$$
\left(\mathbf{C}_{p, q}^{\ell}\right)_{L \times 1}=\left[C_{p, q, k_{1}}^{\ell}, \ldots, C_{p, q, k_{L}}^{\ell}\right]^{T}
$$

The fine correction factors for all data subcarriers can be obtained through linear interpolation [11]. Two consecutive fine correction factors in $L$ pilot tones are used to determine the fine correction factors for other data subcarriers that are located between the $k_{p}$ th and $k_{(p+1)}$ th subcarriers

$$
\begin{array}{r}
C_{p, q, k_{p}+u}^{\ell}=C_{p, q, k_{p}}^{\ell}+\left(\frac{C_{p, q, k_{p}+1}^{\ell}-C_{p, q, k_{p}}^{\ell}}{U}\right) \times u, \\
p=1 \sim L-1, u=1,2, \ldots, U-1 .
\end{array}
$$

The fine channel estimations for all data subcarriers are

$$
\begin{aligned}
& \widehat{H}_{p, q, k_{p}+u}^{\ell} \\
& =C_{p, q, k_{p}+u}^{\ell} \times \widetilde{H}_{p, q, k_{p}+u}^{\ell} \\
& =\left(\frac{\widehat{H}_{p, q, k_{p}}}{\widetilde{H}_{p, q, k_{p}}^{\ell}}\right. \\
& \left.\quad+\left(\frac{\left(\widehat{H}_{p, q, k_{p+1}} / \widetilde{H}_{p, q, k_{p+1}}^{\ell}\right)-\left(\widehat{H}_{p, q, k_{p}} / \widetilde{H}_{p, q, k_{p}}^{\ell}\right)}{U}\right) \times u\right) \\
& \quad \times \widetilde{H}_{p, q, k_{p}+u}^{\ell} \quad \quad p=1 \sim L-1, \quad u=1,2, \ldots, U-1,
\end{aligned}
$$

where $U$ is the number of data subcarriers between two adjacent pilot subcarriers. The fine channel estimations at pilot tones are expressed as

$$
\widehat{H}_{p, q, k_{p}}^{\ell}=C_{p, q, k_{p}}^{\ell} \times \widetilde{H}_{p, q, k_{p}}^{\ell}=\widehat{H}_{p, q, k_{p}}^{\ell}, \quad p=1 \sim L .
$$


The fine channel estimations for those data subcarriers located in the intervals of $\left(0, k_{1}\right)$ and $\left(k_{L}, N-1\right)$ are determined with the fine correction factors $C_{p, q, k_{1}}^{\ell}$ and $C_{p, q, k_{L}}^{\ell}$, respectively. Therefore,

$$
\begin{aligned}
\widehat{H}_{p, q, k_{1}-b}^{\ell} & =C_{p, q, k_{1}-b}^{\ell} \times \widetilde{H}_{p, q, k_{1}-b}^{\ell} \\
& =\frac{\widehat{H}_{p, q, k_{1}}}{\widetilde{H}_{p, q, k_{1}}^{\ell}} \times \widetilde{H}_{p, q, k_{1}-b}^{\ell}, \quad b=1,2, \ldots, k_{1}, \\
\widehat{H}_{p, q, k_{L}+c}^{\ell} & =C_{p, q, k_{L}+c}^{\ell} \times \widetilde{H}_{p, q, k_{L}+c}^{\ell} \\
& =\frac{\widehat{H}_{p, q, k_{L}}}{\widetilde{H}_{p, q, k_{L}}^{\ell}} \times \widetilde{H}_{p, q, k_{L}+c}^{\ell}, \quad c=1,2, \ldots, N-1-k_{L} .
\end{aligned}
$$

Finally, the fine channel estimate vector is given by

$$
\left(\widehat{\mathbf{H}}_{p, q}^{\ell}\right)_{N \times 1}=\operatorname{diag}\left\{\widehat{H}_{p, q, 0}^{\ell}, \widehat{H}_{p, q, 1}^{\ell}, \ldots, \widehat{H}_{p, q, N-1}^{\ell}\right\},
$$

where $\widehat{H}_{p, q, k}^{\ell}, k=0,1, \ldots, N-1$ are obtained from (24), (25), and (26).

Assume that the channel frequency responses $\widehat{\mathbf{H}}_{p, q}$, for $p=1, \ldots, n_{T}$ and $q=1, \ldots, n_{R}$, are known or can be estimated accurately at the receiver, the space-frequency decoder block constructs the even and odd parts of the decision estimate vector $\widehat{\mathbf{X}}$ as

$$
\begin{aligned}
& \widehat{\mathbf{X}}_{e}=\widehat{\mathbf{H}}_{1,1, e}^{*} \mathbf{Y}_{1, e}+\widehat{\mathbf{H}}_{2,1, o} \mathbf{Y}_{1, o}^{*}+\widehat{\mathbf{H}}_{1,2, e}^{*} \mathbf{Y}_{2, e}+\widehat{\mathbf{H}}_{2,2, o} \mathbf{Y}_{2, o}^{*}, \\
& \widehat{\mathbf{X}}_{o}=\widehat{\mathbf{H}}_{2,1, e}^{*} \mathbf{Y}_{1, e}-\widehat{\mathbf{H}}_{1,1, o} \mathbf{Y}_{1, o}^{*}+\widehat{\mathbf{H}}_{2,2, e}^{*} \mathbf{Y}_{2, e}-\widehat{\mathbf{H}}_{1,2, o} \mathbf{Y}_{2, o}^{*} .
\end{aligned}
$$

Substituting (8) into (28) yields

$$
\begin{aligned}
\widehat{\mathbf{X}}_{e}= & \left(\left|\widehat{\mathbf{H}}_{1,1, e}\right|+\left|\widehat{\mathbf{H}}_{2,1, e}\right|+\left|\widehat{\mathbf{H}}_{1,2, e}\right|+\left|\widehat{\mathbf{H}}_{2,2, e}\right|\right) \mathbf{X}_{e} \\
& +\widehat{\mathbf{H}}_{1,1, e}^{*} \mathbf{V}_{1, e}+\widehat{\mathbf{H}}_{2,1, o} \mathbf{V}_{1, o}^{*}+\widehat{\mathbf{H}}_{1,2, e}^{*} \mathbf{V}_{2, e}+\widehat{\mathbf{H}}_{2,2, o} \mathbf{V}_{2, o}^{*}, \\
\widehat{\mathbf{X}}_{o}= & \left(\left|\widehat{\mathbf{H}}_{1,1, o}\right|+\left|\widehat{\mathbf{H}}_{2,1, o}\right|+\left|\widehat{\mathbf{H}}_{1,2, o}\right|+\left|\widehat{\mathbf{H}}_{2,2, o}\right|\right) \mathbf{X}_{o} \\
& +\widehat{\mathbf{H}}_{2,1, e}^{*} \mathbf{V}_{1, e}-\widehat{\mathbf{H}}_{1,1, o} \mathbf{V}_{1, o}^{*}+\widehat{\mathbf{H}}_{2,2, e}^{*} \mathbf{V}_{2, e}-\widehat{\mathbf{H}}_{1,2, o} \mathbf{V}_{2, o}^{*} .
\end{aligned}
$$

\section{Adaptive Path Number Selection Mechanism}

Although MSE in channel frequency response estimate decreases with the path number, under the more serious frequency selective fading channel condition, less selected path number may cause larger error when the interpolation of fine channel frequency response estimation is conducted for all the data subcarriers. Therefore, an adaptive path number selection mechanism is proposed to choose appropriate path number according to the characteristics of time-varying fading channel. The selection procedure of the path number is described as follows. In the first step of the proposed mechanism, the pilot signals in the first OFDM data block

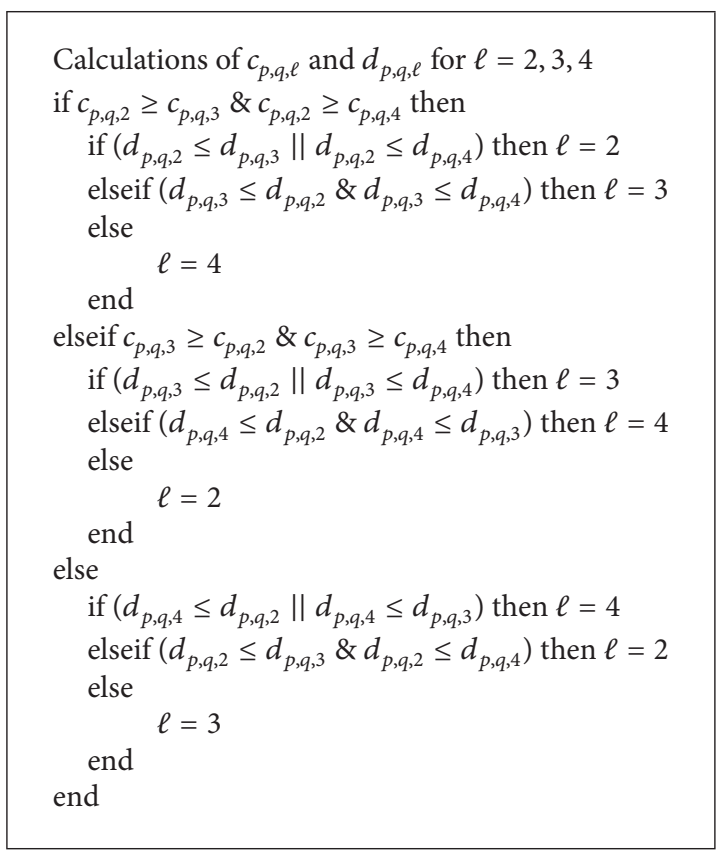

Algorithm 1: Algorithm of adaptive path number selection mechanism for $L=4$.

are used to estimate $\left(\widehat{\mathbf{h}}_{p, q}\right)_{L \times 1}$. In the second step, the preliminary estimates of channel frequency response at pilot tones $\left(\widetilde{\mathbf{H}}_{p, q}^{\ell}\right)_{L \times 1}$ for $\ell=2,3, \ldots, L$ are obtained from (19), and in the third step, the fine correction factors at pilot tones $\left(\mathbf{C}_{p, q}^{\ell}\right)_{L \times 1}$ are obtained from (20), and the fine channel frequency response estimations $\left(\widehat{\mathbf{H}}_{p, q}^{\ell}\right)_{N \times 1}$ are determined by (24), (25), and (26). In the fourth step, the estimated channel frequency transfer function $\left(\widehat{\mathbf{H}}_{p, q}^{\text {long }}\right)_{N \times 1}=\left\{\widehat{H}_{p, q, k}^{\text {long }}, k=\right.$ $0,1, \ldots, N-1\}$ obtained from two continuous long training symbols, which are defined in [9], is compared with the fine channel estimation for path number selection. The difference values between the fine estimations of channel frequency response $\left(\widehat{\mathbf{H}}^{\ell}\right)_{N \times 1}$ and $\left(\widehat{\mathbf{H}}_{p, q}^{\text {long }}\right)_{N \times 1}=\left\{\widehat{H}_{p, q, k}^{\text {long }}, k=0,1, \ldots, N-\right.$ $1\}$ are compared with two times of noise variance of the MIMO-OFDM receiver, respectively. The total number of counts $c_{p, q, \ell}$ which satisfy the condition of $\widehat{H}_{p, q, k}^{\ell}-\widehat{H}_{p, q, k}^{\text {long }}<$ $2 \sigma_{s}^{2}$ is calculated:

$$
\begin{array}{r}
c_{p, q, \ell}=\operatorname{Num}\left(\widehat{H}_{p, q, k}^{\ell}-\widehat{H}_{p, q, k}^{\text {long }}<2 \sigma_{s}^{2}\right. \text { (threshold), } \\
\text { for } k=0,1, \ldots, N-1), \\
\text { for } \ell=2,3, \ldots, L,
\end{array}
$$

where $2 \sigma_{s}^{2}$ is defined as the threshold of $\left(\left(\widehat{\mathbf{H}}_{p, q}^{\ell}\right)_{N \times 1}-\right.$ $\left.\left(\widehat{\mathbf{H}}_{p, q}^{\text {long }}\right)_{N \times 1}\right)$. With reference to the algorithm of adaptive path number selection mechanism listed in Algorithm 1 
TABLE 1: Six-path OFDM channel model.

\begin{tabular}{lcccccccc}
\hline $\begin{array}{l}\text { Path } \\
\text { number }\end{array}$ & $\begin{array}{c}\text { Tap } \\
\text { number }\end{array}$ & $\begin{array}{c}\text { Tap power } \\
(\mathrm{dBm}), K\end{array}$ & $\begin{array}{c}\text { Path power } \\
(\mathrm{dBm})\end{array}$ & $\begin{array}{c}\text { Excess } \\
\text { delay }(n \mathrm{~s})\end{array}$ & $\begin{array}{c}\text { Path } K \\
\text { factor }\end{array}$ & $\begin{array}{c}\text { Freq. offset } \\
\left(f_{d}\right) \text { in Hz }\end{array}$ & $\begin{array}{c}\text { Spectrum half-width } \\
\left(f_{f}\right) \text { in Hz } \mathrm{Hz}\end{array}$ & $\begin{array}{c}\text { Spectrum } \\
\text { shape }\end{array}$ \\
\hline 1 & 1 & 0,102 & -0.0424 & 0 & 0 & 264.6 & - Freq. shift \\
2 & 1 & & -24.892 & 0 & 0 & -75.6 & 476.3 & Rounded \\
3 & 1 & & -21.892 & 0 & 0 & -37.8 & 1001.7 & 1088.6 \\
4 & 2 & -6.5 & -6.5 & 50 & 7.3 & 0 & 1266.3 & Flat \\
5 & 3 & -14.4 & -14.4 & 100 & 4.7 & 0 & 1266.3 & Rounded \\
6 & 4 & -17.5 & -17.5 & 150 & 3.6 & 0 & Rounded \\
\hline
\end{tabular}

for $L=4, d_{p, q, 2}, d_{p, q, 3}, \ldots$, and $d_{p, q, L}$ are defined as the MSE between $\left(\widehat{\mathbf{H}}_{p, q}^{\ell}\right)_{N \times 1}$ and $\left(\widehat{\mathbf{H}}_{p, q}^{\text {long }}\right)_{N \times 1}$ and given by

$$
d_{p, q, \ell}=E\left\{\left\|\widehat{\mathbf{H}}_{p, q, N \times 1}^{\ell}-\widehat{\mathbf{H}}_{p, q, N \times 1}^{\text {long }}\right\|^{2}\right\}, \quad \text { for } \ell=2,3, \ldots, L .
$$

The number of paths can be adaptively selected for the largest $c_{p, q, \ell}$ and the smallest $d_{p, q, \ell}$ in the first OFDM data block or in each of the OFDM data blocks.

\section{Simulation Results}

The function of the proposed adaptive path number selection mechanism is simulated in MIMO-OFDM fading channel. The features for a mobile OFDM system include a bandwidth of $10 \mathrm{MHz}$ and 64 subcarriers, the measured signal interval $T_{m}=$ packet time $=840 \mu \mathrm{sec}$. Each transmitted packet contains 100 OFDM data blocks; the path number selections are conducted at the first data blocks. Four equally spaced pilot subcarriers, which are inserted in the positions of 8th, 24th, 40th, and 56th subcarriers in an OFDM data block, are applied for each of the transmitted OFDM data blocks. The six-path channel model listed in Table 1, where the first three paths have no path delay and the interpath delay time after path three is $50 \mathrm{nsec}$, is employed to simulate mobile OFDM performance, so that $L=4$.

Two-ray Rayleigh fading channel and six-path fading channel with 13 nsec delay spread are used to test the bit error rate (BER) performance of the OFDM transceiver. In Figure 2, two-ray Rayleigh fading channel is used to validate six-path fading channel with 13 nsec delay spread. Figure 2(a) shows the BER performance of OFDM transceiver using 16QAM. Figure 2(b) shows the BER performance of OFDM transceiver using 64-QAM. For demonstration we have chosen $120 \mathrm{~km} / \mathrm{hr}$ and $200 \mathrm{~km} / \mathrm{hr}$; performance for high speed, that is, $200 \mathrm{~km} / \mathrm{hr}$, seems to degrade than the performance for system with less speed, that is, $120 \mathrm{~km} / \mathrm{hr}$, because at higher speed the channel behaves as a fast fading channel and for slower speed channel behaves as a slow fading channel [8]. The test results show that the BER performance of the OFDM transceiver in the high-speed time-varying fading channel will be reduced to less than $10^{-5}$ at the minimum SNR of $12 \mathrm{~dB}$ for $16-\mathrm{QAM}$ and $28 \mathrm{~dB}$ for $64-\mathrm{QAM}$ at $120 \mathrm{Km} / \mathrm{hr}$. For $200 \mathrm{Km} / \mathrm{hr}$ user speed, the performance of 64-QAM OFDM transceiver over six-path fading channel with 13 nsec delay spread degrades to unacceptable levels.

For the purpose of the diversity gain, a simple $2 \times 2$ SFBC is combined with the OFDM system, where the adaptive path number selection mechanism is employed for each transmit-receive antenna pair. Let two frequency-domain data signals at two consecutive subcarriers be encoded using Alamouti code and transmitted from two antennas. Since the channel response for that subcarrier within one SFBC block is stationary, then the maximum-likelihood symbol detector is used to detect the transmitted symbols.

The BER performance of the $2 \times 2$ SFBC-OFDM system in terms of 16-QAM and 64-QAM modulations over the sixpath fading channel with $13 \mathrm{nsec}$ and $26 \mathrm{nsec}$ delay spread is shown in Figures 3 and 4, respectively, where the user speed is set as $200 \mathrm{~km} / \mathrm{h}$. The calculations of $13 \mathrm{nsec}$ and $26 \mathrm{nsec}$ root mean square ( $\mathrm{rms}$ ) delay spread are shown in the appendix. For OFDM system, the channel is frequency nonselective fading if the delay spread is in the range of $(0,20 \mathrm{nsec})$, and the channel is frequency selective fading if the delay spread exceeds $20 \mathrm{nsec}$ [8]. It is observed that the acceptable BER $\left(<10^{-5}\right)$ for QAM modulated MIMOOFDM systems operated in fast-varying fading channels can always be achieved by employing the proposed path number selection mechanism. In Figure 4(a), the BER value of the 16-QAM modulated MIMO-OFDM systems employing the proposed path number selection mechanism over frequency nonselective fast fading channels with $13 \mathrm{nsec}$ delay spread is lower than $\ell=4$ and slightly higher than $\ell=2$ and 3 in the region of interest $\left(\mathrm{BER}<10^{-5}\right)$. The required $E_{b} / N_{0}$ for the acceptable BER is low for all cases. It indicates that the MIMO mode is not necessary to be used for the 16QAM OFDM systems when the delay spread is small. In Figure 4(b), the gain of the 64-QAM modulated MIMOOFDM system employed the proposed adaptive path number selection mechanism in frequency nonselective fast fading channel with 13 nsec delay spread exceeds $1 \mathrm{~dB}$ compared with $\ell=4$, and its BER value is slightly higher than $\ell=2$ and 3 in the region of interest. In frequency nonselective fast fading channel, choosing smaller number of paths can get the smaller mean square error in channel impulse response estimate. Figure 4 shows that the BER value of the QAM modulated MIMO-OFDM systems employing the proposed path number selection mechanism over frequency selective fast fading channels with 26 nsec delay spread is lower than $\ell=2$ and 3 and slightly higher than $\ell=4$ in the region 


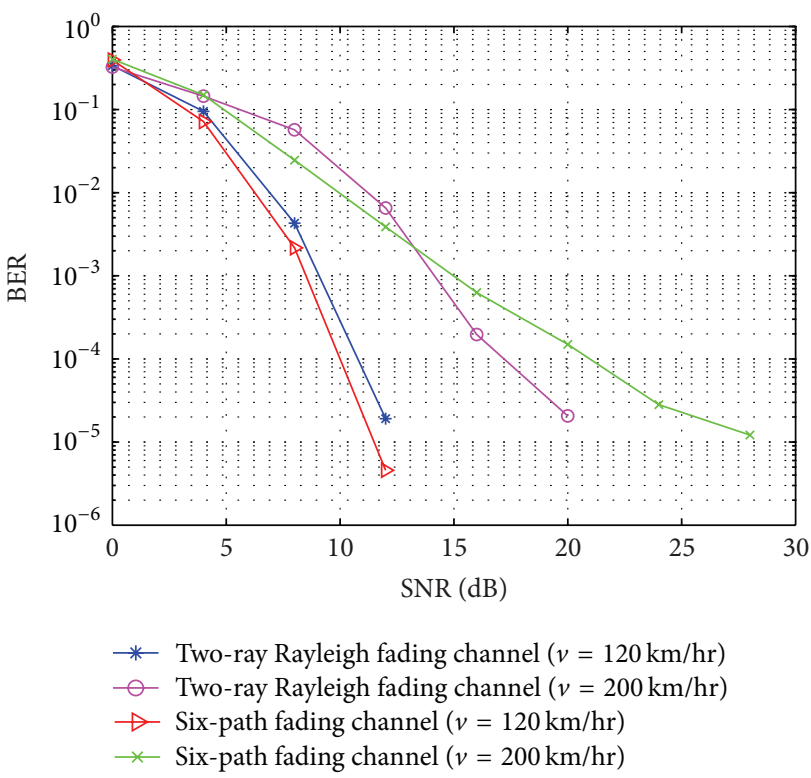

(a)

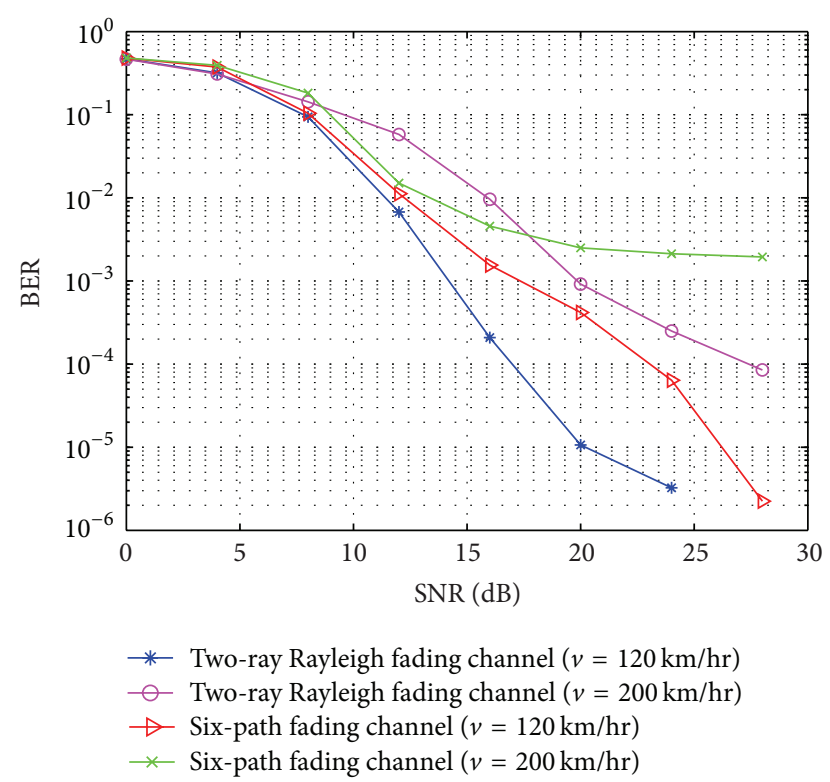

(b)

FIGURE 2: BER of the OFDM transceiver over two-ray Rayleigh fading channel and six-path fading channel with 13 nsec delay spread for (a) 16-QAM and (b) 64-QAM.

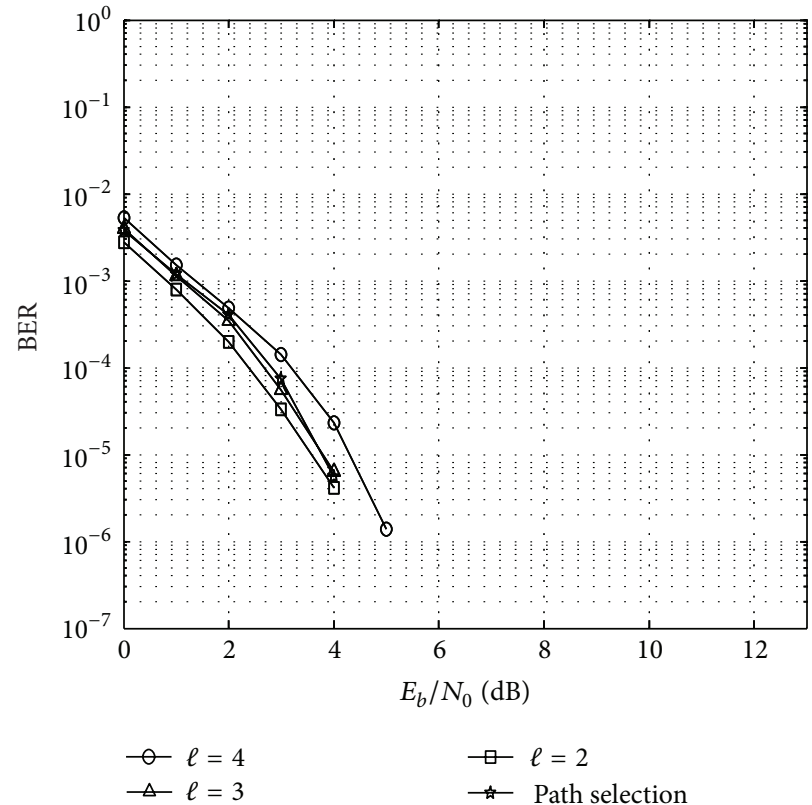

(a)

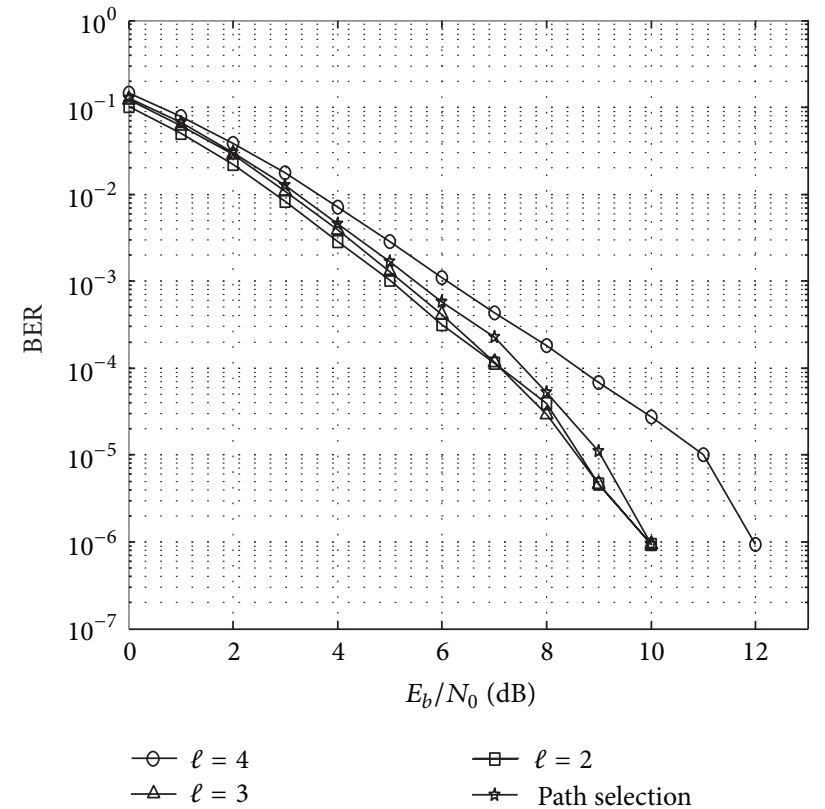

(b)

FIGURE 3: BER performance of $2 \times 2$ SFBC-OFDM system over the six-path fading channel with 13 nsec delay spread for (a) 16-QAM modulation and (b) 64-QAM modulation.

of interest. The error floors of the BER performance appear at an $E_{b} / N_{0}$ of about $8 \mathrm{~dB}$ for 16-QAM (curve $\ell=2$ ) and about $15 \mathrm{~dB}$ for 64-QAM (curves $\ell=2$ and 3), respectively. The frequency selectivity of the multipath fading channel increases with its delay spread. In a frequency selective fast fading channel, the BER of the MIMO-OFDM decreases with an increase of the path number due to more linear interpolation loss are generated by using less path number in frequency selective fast fading channel. By examining Figures 3 and 4 , it is concluded that the pilot-based channel estimate using the proposed path number selection mechanism at the first data block can satisfy the OFDM system performance requirements under different operating conditions of timevarying fast fading channels. 


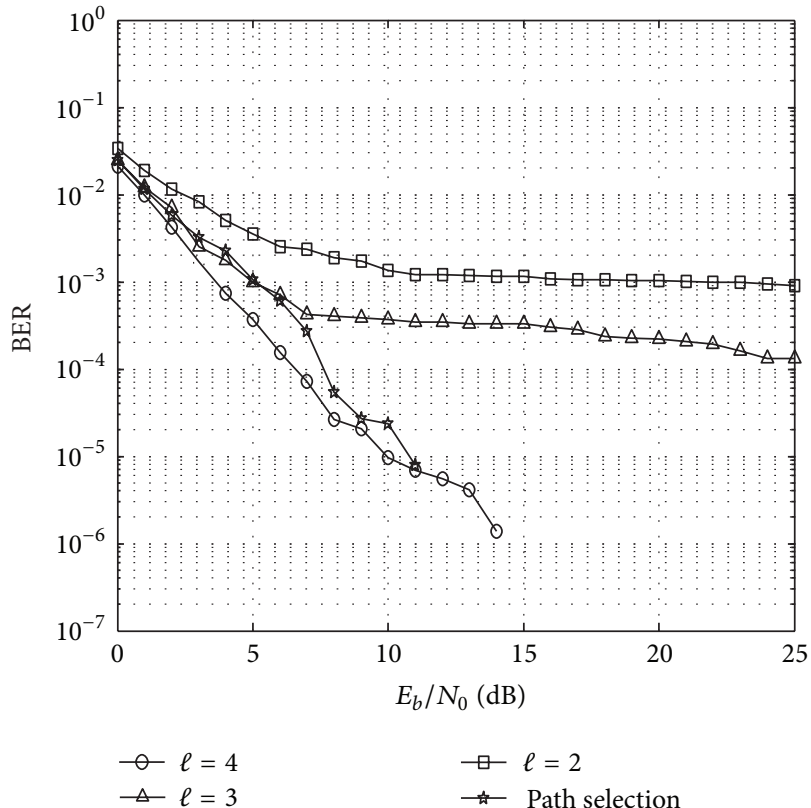

(a)

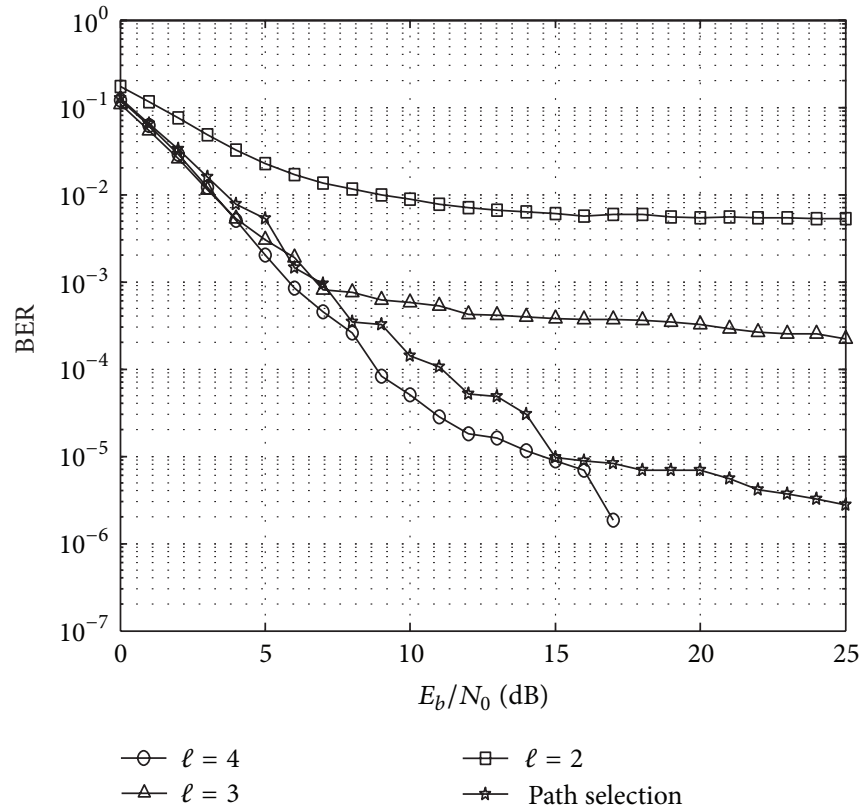

(b)

FIGURE 4: BER performance of $2 \times 2$ SFBC-OFDM system over the six-path fading channel with 26 nsec delay spread for (a) 16-QAM modulation and (b) 64-QAM modulation.

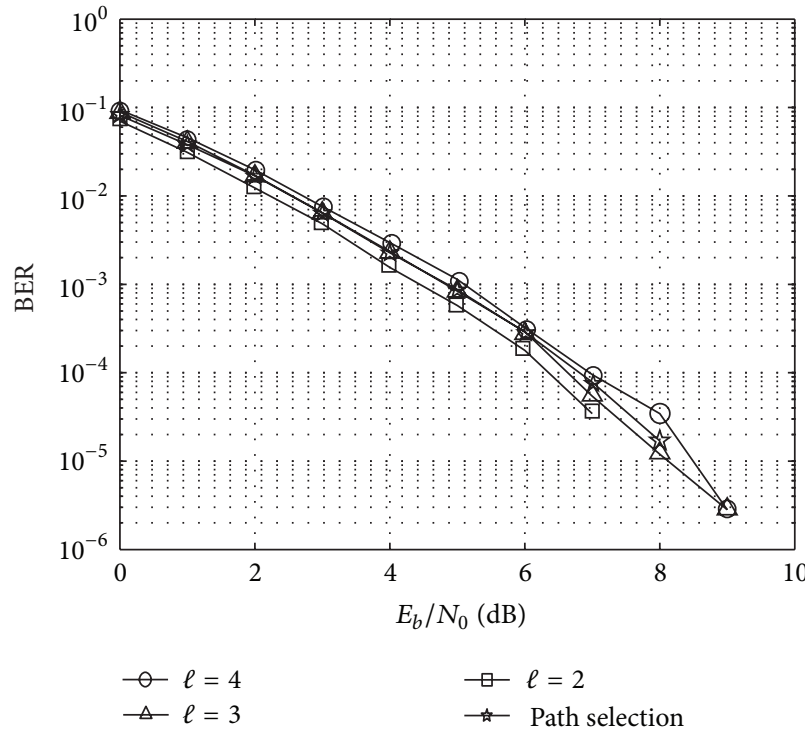

(a)

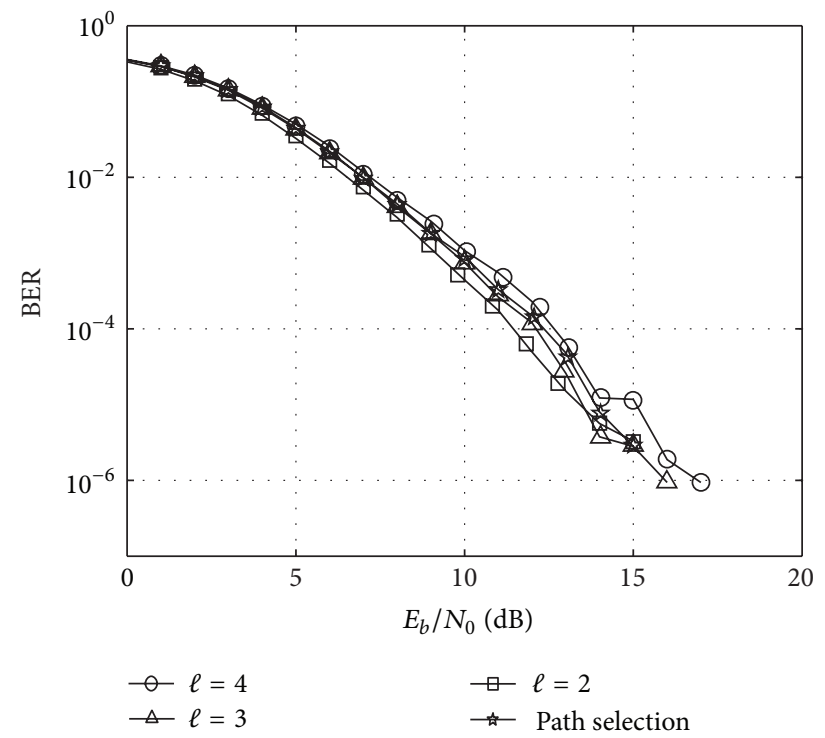

(b)

FIGURE 5: BER performance of OFDM system over the six-path fading channel with 13 nsec delay spread for (a) 16-QAM modulation and (b) 64-QAM modulation.

In Figure 5, the BER value of the QAM modulated OFDM system employing the proposed path selection mechanism over time-varying fading channels with $13 \mathrm{~ns}$ delay spread is lower than $\ell=4$ and slightly higher than $\ell=2$ and 3 .

Figure 6 shows that the BER value of the QAM modulated OFDM systems employing the proposed path selection mechanism over time-varying fading channels with $26 \mathrm{~ns}$ delay spread is lower than $\ell=2$ and 3 and slightly higher than $\ell=4$. The frequency selectivity of the multipath fading channel increases with its delay spread.

\section{Conclusions}

An adaptive path number selection mechanism is proposed to improve the accuracy of the pilot-based channel estimation approach for QAM modulated MIMO-OFDM systems 


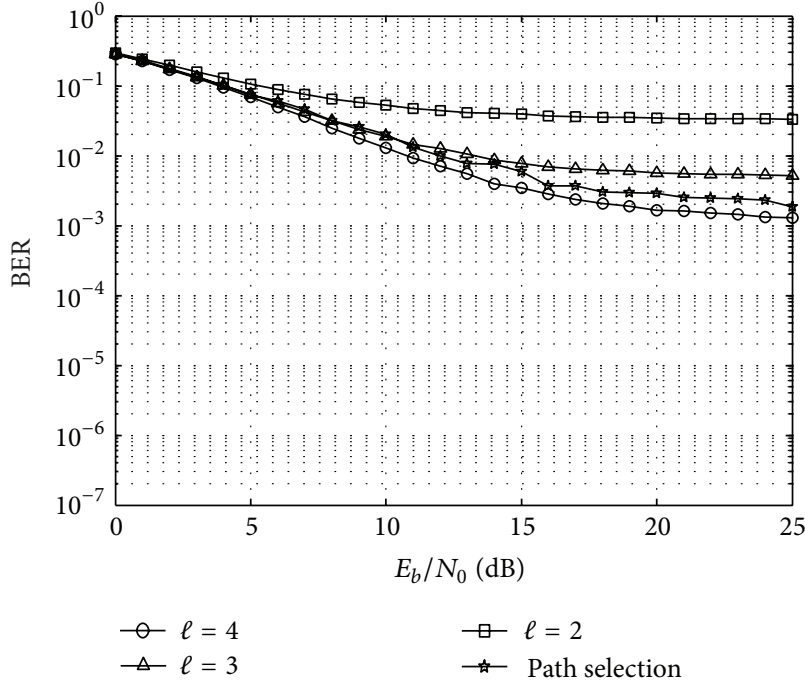

(a)

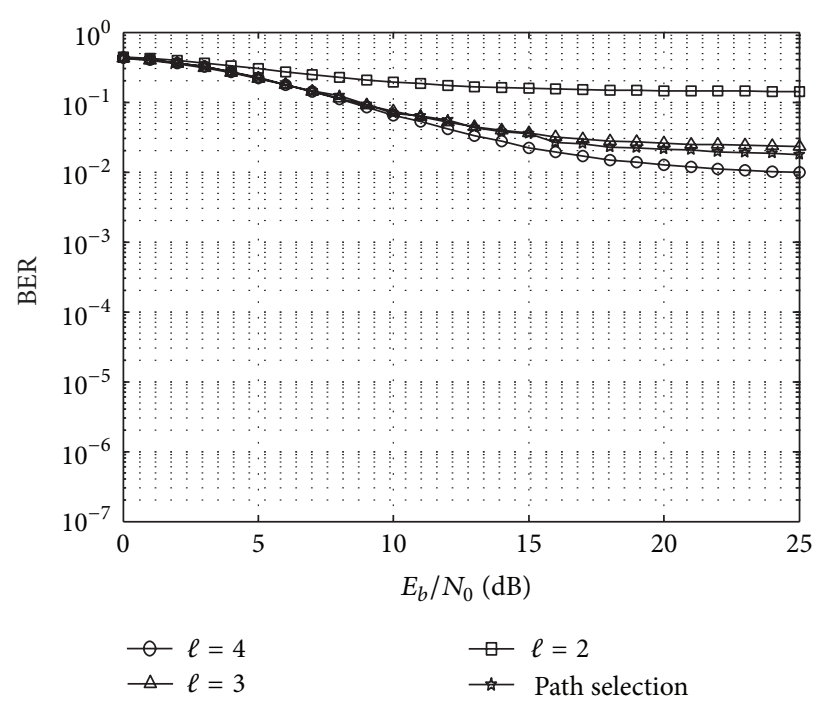

(b)

FIGURE 6: BER performance of OFDM system over the six-path fading channel with 26 nsec delay spread for (a) 16-QAM modulation and (b) 64-QAM modulation.

operating in time-varying fast fading channels. The fine correction factors are derived. It is demonstrated that the number of paths is scalable and adaptively changed with the characteristics of time-varying MIMO-OFDM fading channels to provide a suboptimum BER performance for QAM modulated $2 \times 2$ SFBC-OFDM systems operated either in frequency nonselective fast fading or in frequency selective fast fading channels.

\section{Appendix}

\section{Calculations of Delay Spread 26 nsec and $13 \mathrm{nsec}[8]$}

The fading channel generator at the $i$ th path consists of two nonline-of-sight (NLOS) branches and a LOS branch. In NLOS branch, two independent and identically distributed (iid) Gaussian signal sources are connected to identical Doppler filters. The channel weighing factor $w_{i}\left[k^{\prime}\right]$ is determined by

$$
w_{i}[k]=P_{i}\left(s_{i-\mathrm{NLOS}}[k]+s_{i \_\mathrm{LOS}}[k]\right), \quad k=1,2, \ldots N,
$$

where the output of NLOS branch at the $i$ th path in the fading channel weighing generator is

$$
s_{i-\mathrm{NLOS}}[k]=y_{i}[k] \times\left(\frac{1}{\sqrt{1+k_{i}}}\right), \quad k=1,2, \ldots, N,
$$

where

$$
\begin{array}{r}
y_{i}[k]=\left(\sum_{l=-\infty}^{\infty} x_{1}[l] \widehat{h}_{i}[k-l]\right)+j\left(\sum_{l=-\infty}^{\infty} x_{2}[l] \widehat{h}_{i}[k-l]\right) \\
k=1,2, \ldots N .
\end{array}
$$

$x_{1}$ and $x_{2}$ are independent Gaussian functions, and $\widehat{h}_{i}$ is impulse response of Doppler filter. The output of LOS branch at $i$ th path in the fading channel weighing generator is

$$
\begin{array}{r}
s_{i_{\mathrm{LOS}}}[k]=\sqrt{\frac{k_{i}}{1+k_{i}}} \times \exp \left(j 2 \pi\left(f_{d_{i}} \cos \theta_{i}+f_{o_{i}}\right) \frac{k}{f_{s, \mathrm{dop}}}\right) \\
k=1,2, \ldots N .
\end{array}
$$

Furthermore, $w_{i}[k]$ is interpolated by factor $I$ to get the sequence $v_{i}\left[k^{\prime}\right]$ with sampling rate $f_{\text {sig }}=I \times f_{s, \text { dop }}$ :

$$
v_{i}\left[k^{\prime}\right]= \begin{cases}w_{i}\left[\frac{k^{\prime}}{I}\right] & k^{\prime}=I, 2 I, \ldots, N I \\ 0 & \text { otherwise. }\end{cases}
$$

The image signal of $v_{i}\left[k^{\prime}\right]$ is removed by an interpolation low pass filter with unit impulse response $h_{I}\left[k^{\prime}\right]$. The interpolated fading envelop signal $r_{i}\left[k^{\prime}\right]$ is stored in the register bank as

$$
r_{i}\left[k^{\prime}\right]=\sum_{l=-\infty}^{\infty} v_{i}[l] h_{I}[k-l], \quad k^{\prime}=1,2, \ldots, N I,
$$


where the sampling interval of $r_{i}\left[k^{\prime}\right]$ is

$$
\Delta t=\frac{1}{f_{\text {sig }}} \text {. }
$$

The parameters listed in Table 1 represent for rms delay spread $\tau_{\text {rms }}=23 \mathrm{nsec}$. As an example steps involved in the calculation of $\tau_{\text {rms }}=23 \mathrm{nsec}$ using the values in Table 1 will be shown. From Table 1 , the Tap powers $\left(P_{i}\right)$ are $0 \mathrm{~dB}$, $-6.5 \mathrm{~dB},-14.4 \mathrm{~dB}$, and $-17.5 \mathrm{~dB}$ at Excess delay $0 \mathrm{nsec}, 50 \mathrm{nsec}$, $100 \mathrm{nsec}$, and $150 \mathrm{nsec}$, respectively. We first normalize these Tap powers to get values $0.9751 \mathrm{~W}, 0.2183 \mathrm{~W}, 0.0354 \mathrm{~W}$, and $0.0173 \mathrm{~W}$ at Excess delay $0 \mathrm{nsec}, 50 \mathrm{nsec}, 100 \mathrm{nsec}$, and 150 nsec respectively.

Normalized tap delay powers are weighed using weighing factor $w_{i}\left[k^{\prime}\right]$. As an example, if weighing factors were found to be $0.7099-0.4263 i,-0.6830-0.3211 i,-0.393-0.4039 i$, and $-0.0443-0.0841 i$ for excess delay at $0 \mathrm{~ns}, 50 \mathrm{~ns}, 100 \mathrm{~ns}$, and 150 ns, respectively. Taking the absolute values for the tap delay power and the weighing factor we get final tap powers $0.8074,0.1648,0.0200$, and 0.0016 at Excess delay 0 nsec, $50 \mathrm{nsec}, 100 \mathrm{nsec}$ and $150 \mathrm{nsec}$, respectively thus, calculating rms delay spread using (A.8) [12]:

$$
\tau_{\mathrm{drms}}=\sqrt{\overline{\tau^{2}}-(\bar{\tau})^{2}}
$$

where the mean excess delay $\bar{\tau}$ is defined as

$$
\bar{\tau}=\frac{\sum_{k} a_{k}^{2} \tau_{k}}{\sum_{k} a_{k}^{2}}=\frac{\sum_{k} P\left(\tau_{k}\right) \tau_{k}}{\sum_{k} P\left(\tau_{k}\right)}
$$

and $\overline{\tau^{2}}$ is defined as

$$
\overline{\tau^{2}}=\frac{\sum_{k} a_{k}^{2} \tau_{k}^{2}}{\sum_{k} a_{k}^{2}}=\frac{\sum_{k} P\left(\tau_{k}\right) \tau_{k}^{2}}{\sum_{k} P\left(\tau_{k}\right)} .
$$

Similarly, tap powers $0 \mathrm{db},-18.5 \mathrm{~dB},-21.4 \mathrm{~dB}$, and $-24.5 \mathrm{~dB}$ for Excess delay $0 \mathrm{~ns}, 50 \mathrm{~ns}, 100 \mathrm{~ns}$, and $150 \mathrm{~ns}$, respectively, for $\tau_{\text {rms }}=13 \mathrm{nsec}$ and tap powers $0 \mathrm{db},-6.5 \mathrm{~dB},-7.5 \mathrm{~dB}$, and $-8.5 \mathrm{~dB}$ for Excess delay $0 \mathrm{~ns}, 50 \mathrm{~ns}, 100 \mathrm{~ns}$, and $150 \mathrm{~ns}$, respectively, for $\tau_{\text {rms }}=26 \mathrm{nsec}$.

\section{Acknowledgment}

This work is supported in part by research grants from National Science Council, Taiwan (NSC 102-2218-E-155-001).

\section{References}

[1] S. R. Saunders and A. A. Zavala, Antennas and Propagation for Wireless Communication Systems, John Wiley \& Sons, 2nd edition, 2007.

[2] T. D. Chiueh and P. Y. Tsai, OFDM Baseband Receiver Design for Wireless Communications, John Wiley \& Sons, 2007.

[3] J. Chen, Y. Tang, and S. Li, "Analysis and optimization of pilot-symbol-assisted M-OAM for OFDM systems," Wireless Communications and Mobile Computing, vol. 5, no. 1, pp. 15-22, 2005.
[4] O. Simeone, Y. Bar-Ness, and U. Spagnolini, "Pilot-based channel estimation for OFDM systems by tracking the delaysubspace," IEEE Transactions on Wireless Communications, vol. 3, no. 1, pp. 315-325, 2004.

[5] M. Torabi, S. Aïssa, and M. R. Soleymani, "On the BER performance of space-frequency block coded OFDM systems in fading MIMO channels," IEEE Transactions on Wireless Communications, vol. 6, no. 4, pp. 1366-1373, 2007.

[6] I. Cosovic and G. Auer, "Capacity of MIMO-OFDM with pilot-aided channel estimation," Eurasip Journal on Wireless Communications and Networking, vol. 2007, Article ID 32460, 2007.

[7] J. Kim, R. W. Heath Jr., and E. J. Powers, "Receiver designs for Alamouti coded OFDM systems in fast fading channels," IEEE Transactions on Wireless Communications, vol. 4, no. 2, pp. 550559, 2005.

[8] J. Mar, C.-C. Kuo, Y.-R. Lin, and T.-H. Lung, "Design of software-defined radio channel simulator for wireless communications: case study with DSRC and UWB channels," IEEE Transactions on Instrumentation and Measurement, vol. 58, no. 8, pp. 2755-2766, 2009.

[9] "Standard Specification for Telecommunications and Information Exchange Between Roadside and Vehicle System5Ghz Band Dedicated Short Range Communications (DSRC) Medium Access Control (MAC) and Physical Layer (PHY) Specifications," IEEE 802, December 2005.

[10] R. Negi and J. Cioffi, "Pilot tone selection for channel estimation in a mobile ofdm system," IEEE Transactions on Consumer Electronics, vol. 44, no. 3, pp. 1122-1128, 1998.

[11] J. Rinne and M. Renfors, "Pilot spacing in Orthogonal Frequency Division Multiplexing systems on practical channels," IEEE Transactions on Consumer Electronics, vol. 42, no. 4, pp. 959-962, 1996.

[12] T. S. Rappaport, Wireless Communications: Principles and Practice, Prentice Hall, 2nd edition, 2002. 

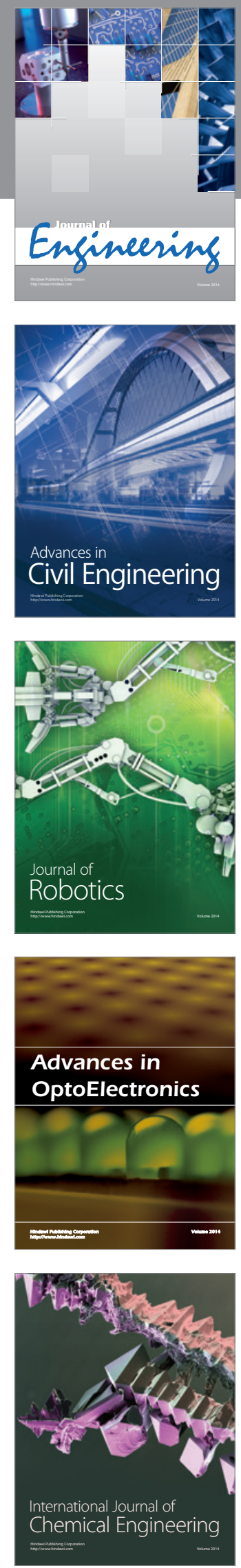

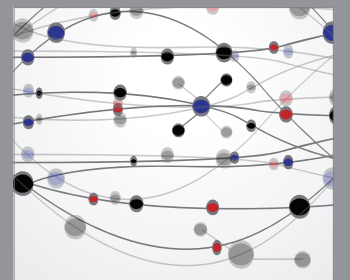

The Scientific World Journal
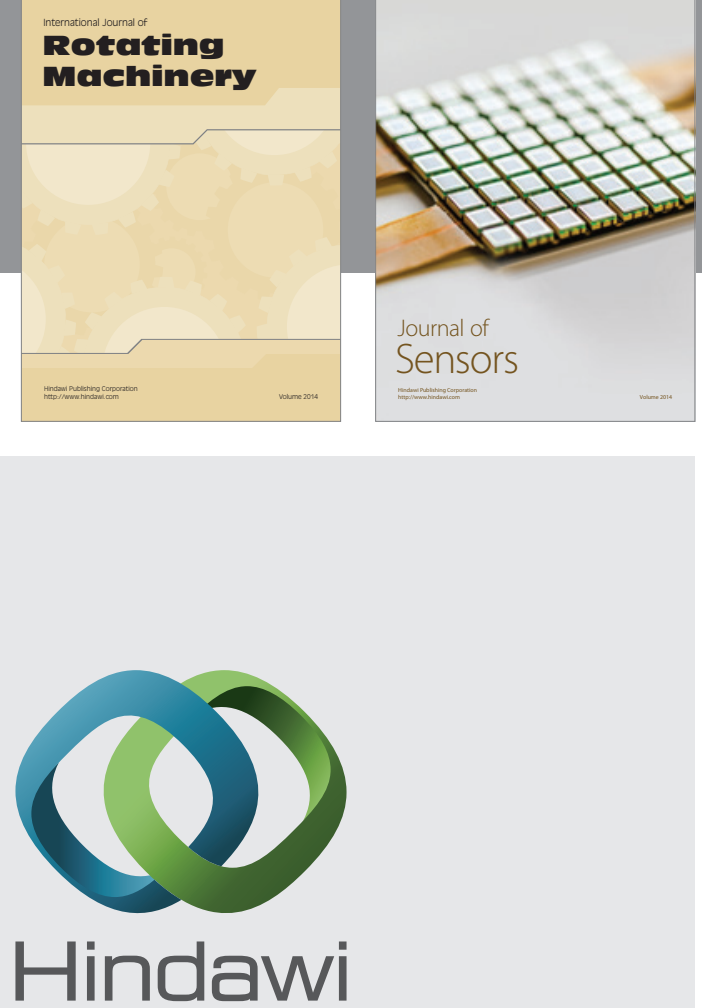

Submit your manuscripts at http://www.hindawi.com
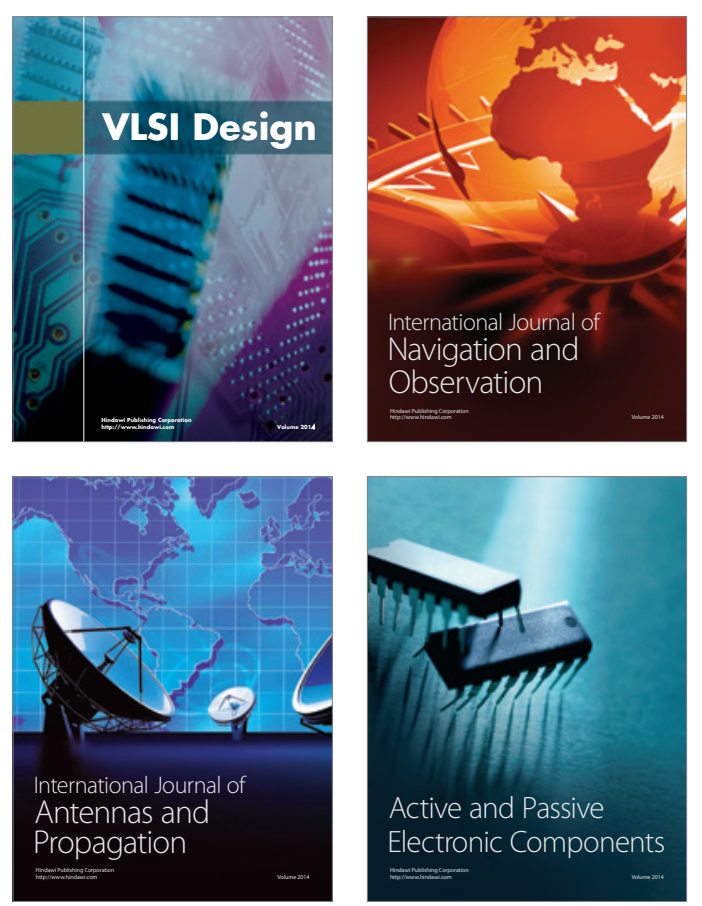
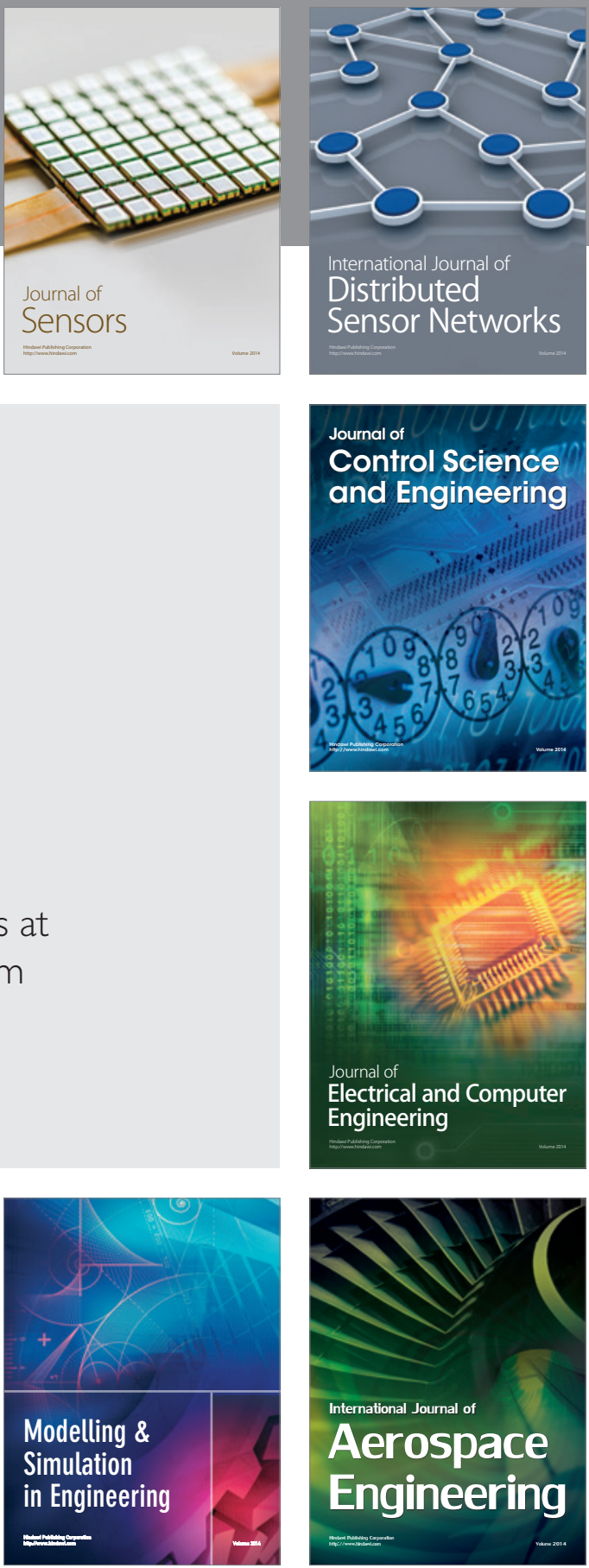

Journal of

Control Science

and Engineering
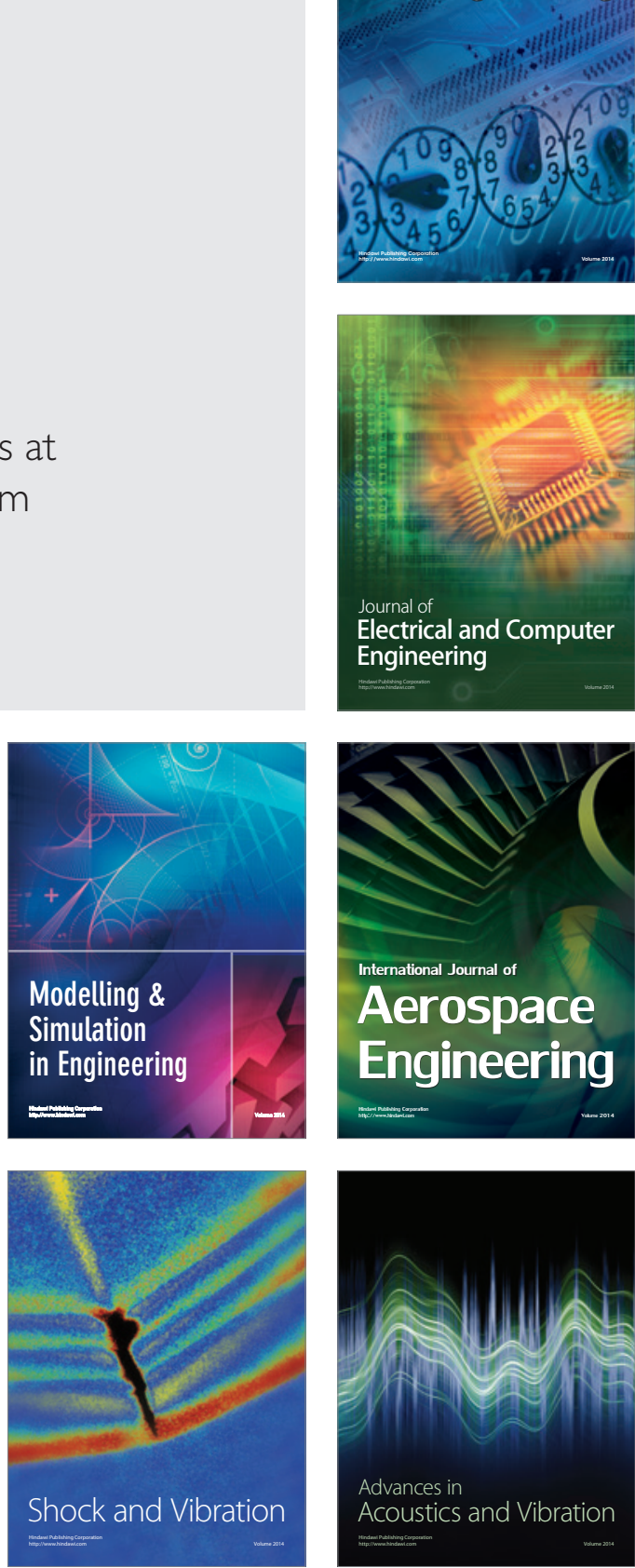